\title{
ACTION DES ECTOPARASITES SUR LES CULICIDES
}

\author{
Par N. LEON
}

Les ectoparasites qui vivent sur les culicides sont généralement des larves d'acariens, dont le nombre peut devenir parfois assez considérable, à en juger par la photographie que reproduit la figure 1. En s'aidant de leur rostre, ces ectoparasites s'introduisent dans le tégument et sucent le sang du culicide. Nous avons encore observé que les culicides n'étaient pas seulement parasités par les acariens, mais aussi par de petites espèces de Culicoüdes ; ces insectes, on le sait, ont un appareil buccal très puissant et la piqûre, chez l'homme, en est très douloureuse. Ces parasites enfoncent de

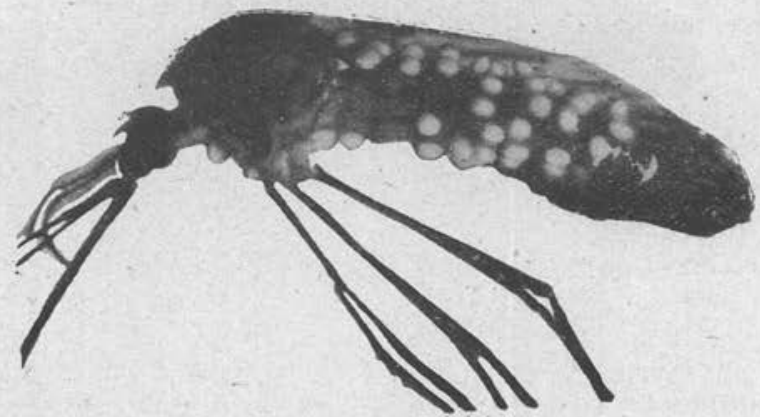

Fig. 1. - Femelle d'Anopheles, sur le corps de laquelle sont fixées plusieurs larves d'acariens.

même leur rostre dans le tégument de l'abdomen et lorsque l'estomac du culicide est gonflé par le sang d'un vertébré, la trompe du parasite pénètre dans la paroi de l'estomac et se met en devoir de sucer le sang du vertébré dont les parasites en question se nourissent.

Les Culicoïdes ne possèdent pas seulement cette action spoliatrice, ils peuvent aussi inoculer des germes infectieux. Dans le cas que nous avons observé, ils ont ouvert la porte d'entrée aux spores de moisissures qui, sans eux, n'auraient pu franchir les barrières épithéliales que l'organisme des culicides oppose à l'infection. Ils peuvent produire des tumeurs mycosiques et des hernies stomacales.

Annales de Parasitologie, $\mathrm{T}, \mathrm{II}, \mathrm{N}^{\circ} 3,-$ Juillet 1924 , p. 211. 
1. Tumeur mycosique. - Chez une femelle d'Anopheles maculipennis, sur la partie gauche de l'abdomen, à la hauteur du troisième segment antérieur, nous avons remarqué une tumeur un peu plus volumineuse que la tête de l'Anopheles et qui communiquait avec l'intérieur de l'abdomen par un orifice de la cuticule. La tumeur provient de la blessure faite dans la cuticule par un Culocoides ; il s'est établi, sur cette blessure, une spore de champignon, lequel a provoqué la tuméfaction réactionnelle.

La paroi de la tumeur est constituée par une membrane épaisse garnie, à la surface, de poils cuticulaires. A l'intérieur de la tumeur, on trouve un coagulum avec des filaments mycéliens, des spores, des amibocites et des trachées. Les filaments mycéliens sont cloi-

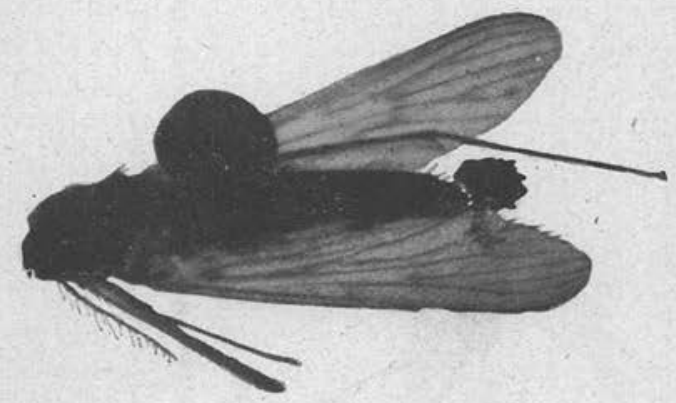

Fig. 2. - Femelle d'Anopheles affectée d'une hernie stomacale.

sonnés, incolores, extrêmement ramifiés et enchevêtrées. Les spores, en groupes, se développent à l'extrémité des filaments, sous forme de glomérules ou de longs filaments.

$2^{\circ}$ Hernie stomacale. - Chez une autre femelle d'Anopheles maculipennis (fig. 2) et également sur la partie gauche de l'abdomen, nous remarquâmes une tumeur présentant un volume quatre fois environ plus gros qu'une tête de femelle d'Anopheles. La tumeur était située entre le deuxième et le troisième segment abdominal antérieur et était comprise entre le pleurite et le tergite; la tumeur est reliée à l'abdomen par un pédoncule. Nous avons encore trouvé une tumeur abdominale semblable chez une autre femelle d'Anopheles. Cette tumeur était remplie de sang d'oiseau, ce qui, dès cette époque, nous a fait supposer que nous nous trouvions en présence d'une hernie stomacale. Aussi avons-nous tout d'abord photographié ce second exemplaire (fig. 2) et n'avons-nous étudié qu'après cette tumeur, d'après les coupes que nous en avons faites. Il s'agit véritablement ici d'une hernie stomacale qui, à 
notre avis, a pris naissance dans la plaie faite sur l'abdomen par un Culicoïdes.

Lorsque l'estomac de l'Anopheles est rempli de sang, l'abdomen de l'insecte est gonflé, la paroi de l'estomac arrive en contact direct avec la face interne du tégument abdominal, de sorte que, si le Culicoïdes pique juste à ce moment, son rostre pénètre dans l'estomac et suce le sang à son tour; par contre, si le Culicoïdes pique l'Anopheles lorsque celui-ci est affamé, c'est-à-dire au moment où l'estomac est vide, comme dans le cas présent, l'estomac occupant la ligne médiane de l'abdomen, le rostre du Culicö̈des ne pourra naturellement pas atteindre l'estomac. Dans ces conditions, le Culicoïdes ne pouvant pas se nourrir retirera son rostre de la blessure et s'envolera. Cependant, quelque temps plus tard l'Anopheles, trouvant l'occasion de sucer le sang d'un vertébré quelconque, son estomac se gonfle sous l'afflux du sang, jusqu'à ce qu'il remplisse la cavité abdominale et qu'il appuie sur la paroi interne de la cavité, donnant ainsi naissance à une évagination qui sor tira à la surface de l'abdomen par la blessure qu'aura faite le Culicoïdes. C'est de cette manière que se forme la hernie stomacale.

La tumeur, ou pour mieux dire la hernie stomacale, est constituée par une paroi et un contenu. La paroi affecte la même strue. ture histologique que l'estomac, étant donné que c'est une simple évagination de celui-ci. Cette paroi est formée de trois tuniques : $1^{\circ}$ la tunique externe, formée par le tissu conjonctif ; $2^{\circ}$ la tunique moyenne, ou tunique musculeuse, beaucoup plus épaisse que la précédente et qui est composée de deux couches de fibres musculaires : la couche interne, constituée par des fibres circulaires qui ne paraissent pas être continues et la couche externe qui est formée par des fibres longitudinales ; $3^{\circ}$ la tunique interne ou muqueuse, constituée par une couche de cellules cylindriques, granuleuses, possédant un noyau sphérique.

Le contenu consiste en un caillot de sang. Dans notre premier exemplaire, le caillot était formé de globules de sang d'oiseau; dans le second exemplaire le caillot était formé de sang humain. La majeure partie des globules rouges sont normaux; certains d'entre eux sont hémolysés. On distingue aussi de petits amas de pigment.

Laboraloire de Parasilologie de la Faculté de médecine de Jassy (Roumanie). 